\title{
Case Report of Prenatal Diagnosis of Klinefelter Syndrome Based on Ultrasound Screening
}

\section{ART ICLE INF O}

\section{Article Type}

Case Report

\section{Authors}

Haddadi N.* MD

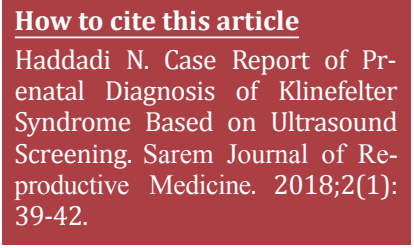

*Sarem Fertility \& Infertility Research Center (SAFIR), Sarem Women's Hospital, Tehran, Iran

\section{Correspondence}

Address: Sarem Women's Hospital, Basij Square, Phase 3, Ekbatan Town, Tehran, Iran. Postal Code: 1396956111

Phone: +98 (21) 44670888

Fax: +98 (21) 44670432

n_hadadi@yahoo.com

\section{Article History}

Received: August 12, 2016

Accepted: January10, 2017

ePublished: February 15, 2018

\section{A B S T R A C T}

Patient Information About one-third of embryos with Nuchal Thickening (NT) have chromosomal abnormalities. One of these abnormalities is Klinefelter syndrome (47XXY). The aim of this case report was to indicate the importance of prenatal diagnosis with consideration of anomalies reports in both screenings of the first and second trimester of pregnancy as well as the importance of NT and the need for further chromosomal studies in these conditions.

A 29-year-old woman with the first pregnancy was referred to Screening Clinic of Sarem Hospital for the first stage of aneuploidy screening in gestational age of 13 weeks and 2 days. The screening results of the first trimester were normal and in the second screening, the Quad marker testing (four tests of Inhibin A, Free $\beta$-hCG, AFP, and UE3) was conducted in gestational age of 16 weeks; the risk of Fetal Neural Tube Defects and Down syndrome were reported again in the low-risk range. Amniocentesis was asked for the patient for a definitive diagnosis because of the high NT range. In the review and analysis of amniotic fluid cells, karyotype was reported as 47XXY (Klinefelter syndrome).

Conclusion In aneuploidy screening, only estimating the risk of common trisomies of 13, 18, and 21 is not the case. NT amount must be examined separately. The prenatal diagnosis of these disorders is effective in planning and future prognosis of pregnancy. Klinefelter syndrome is one of those chromosomal abnormalities, which occurs as a XXY karyotype with an outbreak of 1 in 10,000 in boys, and, unfortunately, it is not diagnosed until the puberty after manifesting itself as a demonstration of hypogonadism. Early diagnosis and alternative hormone therapy can make natural maturation process in these cases.

Keywords Aneuploidy; Screening; Klinefelter Syndrome; First Trimester; Nuchal Translucency Mesurement

\section{I T A T I O N L I N KS}

[1] The 11-13+ 6 Weeks Scan [2] Non-invasive screening methods of down syndrome through maternal circulation [3] Nuchal translucency thresholds in prenatal screening for Down syndrome and trisomy 18 [4] Technical standards and guidelines: Prenatal screening for Down syndrome that includes first-trimester biochemistry and/or ultrasound measurements [5] Fetal chromosomal abnormalities prevent programs down syndrome [6] Prenatal diagnosis of genetic diseases [7] Screening of chromosomal abnormalities in 1st and 2nd trimester of pregnancy: The role of fetal echocardiographic genetic sonography [8] Screening for fetal aneuploidy and neural tube defects [9] Ultrasound in prenatal diagnosis of triploidy and Turner syndrome [10] Fetal nuchal translucency screening in 12 495 pregnancies in Sardinia [11] Klinefelter syndrome: the commonest form of hypogonadism, but often overlooked or untreated. Psychological and neurological problems [12] Prenatally detected double trisomy: Klinefelter and Down syndrome [13] Double aneuploidy 48,XXY,+21 in a fetus with congenital abnormalities [14] Double aneuploidy 48, XXY,+ 21 associated with a aongenital aeart aefect in a neonate balkan [15] Klinefelter syndrome diagnosed by prenatal screening tests in high-risk groups [16] Trends in the utilization of invasive prenatal diagnosis in the Netherlands during 20002009 
فقرات קشت گردن است، زمانى كه CRL بين 0 ع تا عـميلىمتر

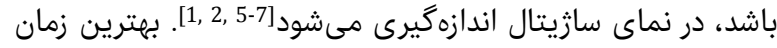

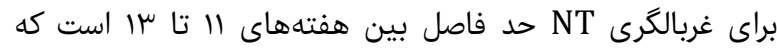

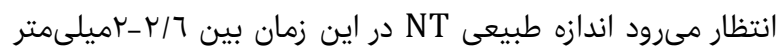

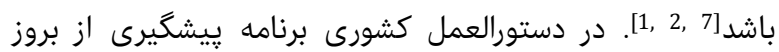

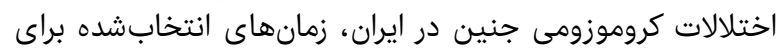

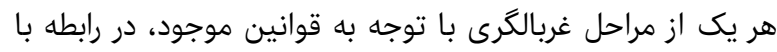

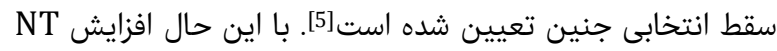

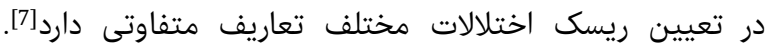

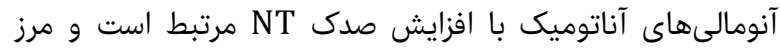
NT

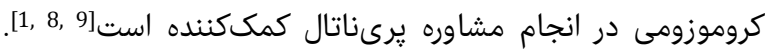

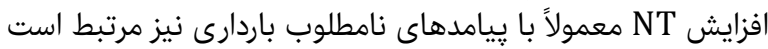

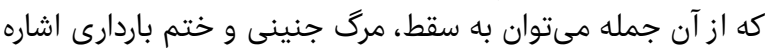

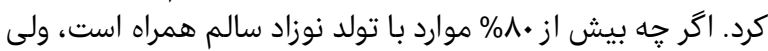

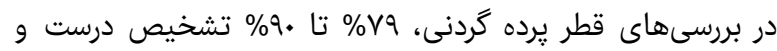

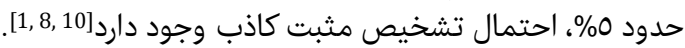

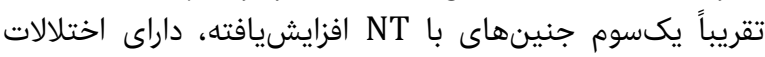

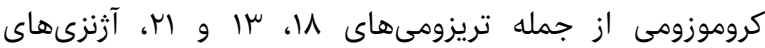

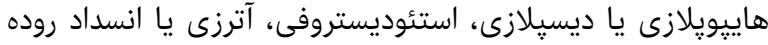

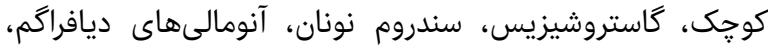

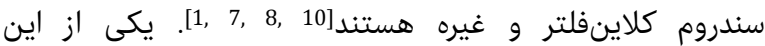

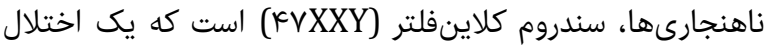

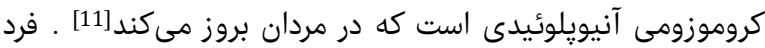

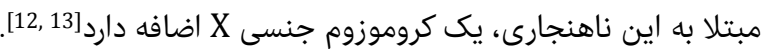

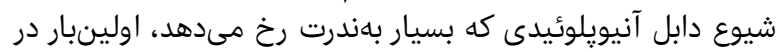

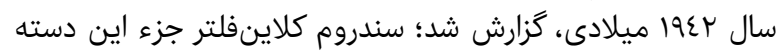

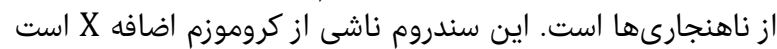

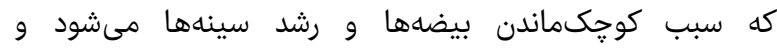

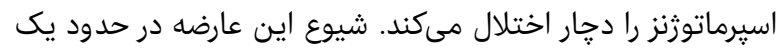

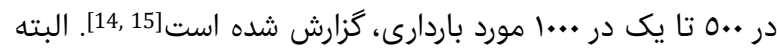

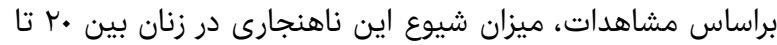

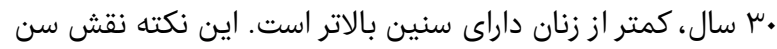

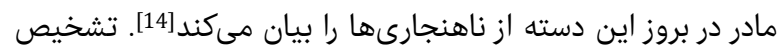

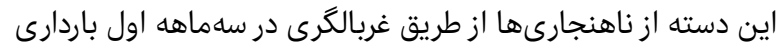

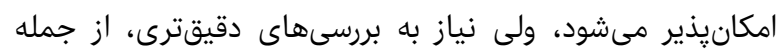

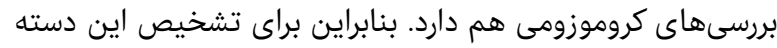

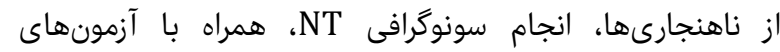

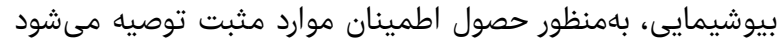

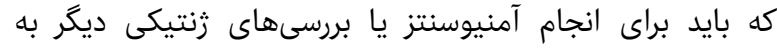
آزمايشكاه زنتنيك ارجاع داده شوند.

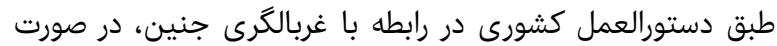

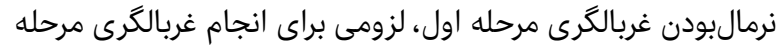

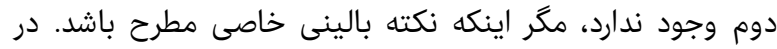

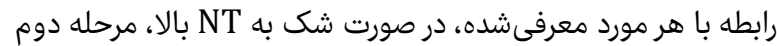

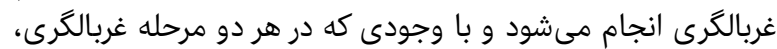

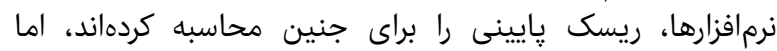

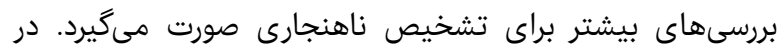

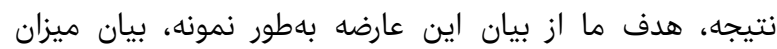

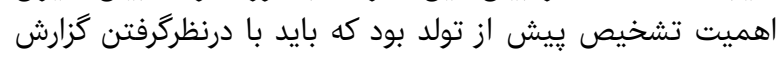

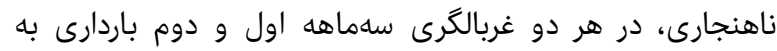

\section{تشخيص ييش از تولد إن سندروم كلاينفلتر بر

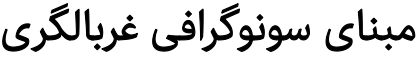

نكين حدادى" MD مركز تحقيقات بارورى و نابارورى صارم، بيمارستان فوق تخصصى صارم، تهران، ايران

جكيده

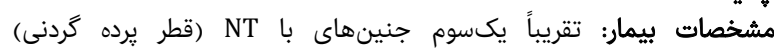

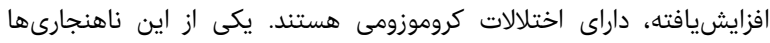

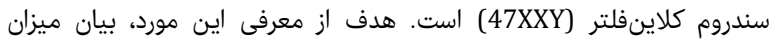

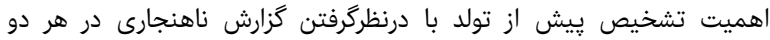

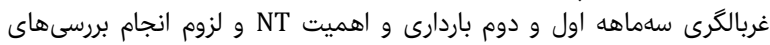

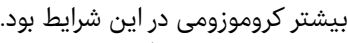

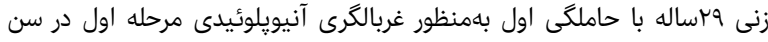

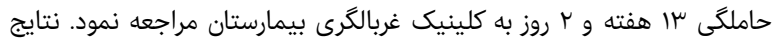

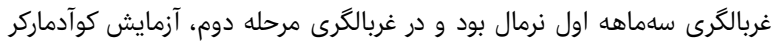

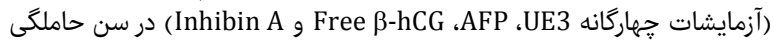

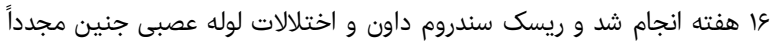

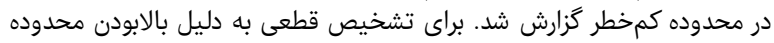

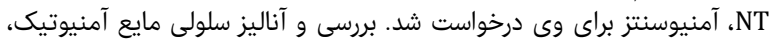
كاريوتايي را بهصورت NTXXY

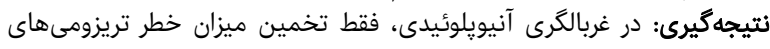

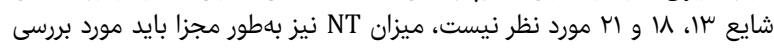

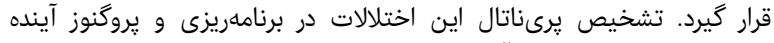

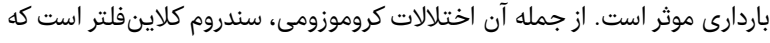

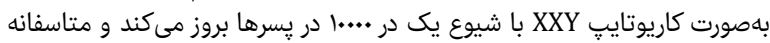

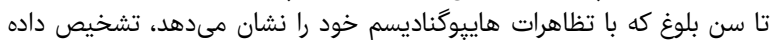

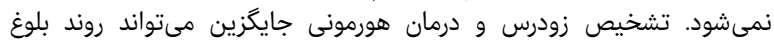

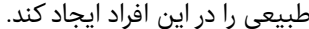

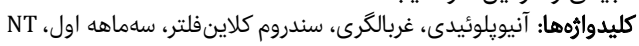

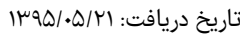

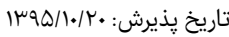

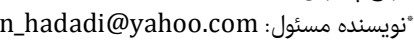

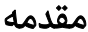

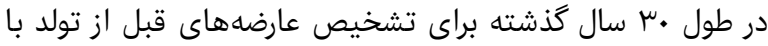

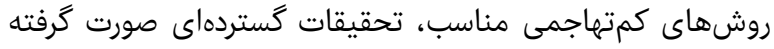

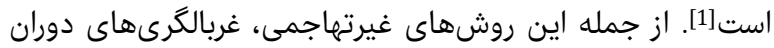

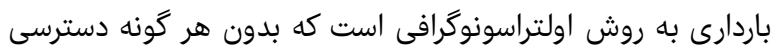

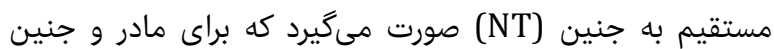

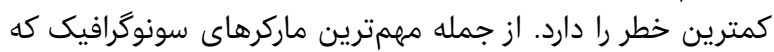

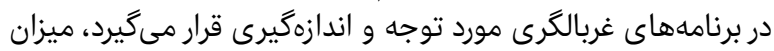

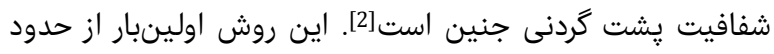

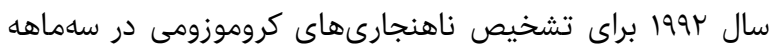

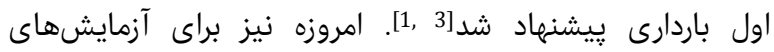

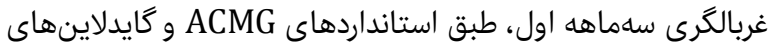
Free مربوط به آن، آزمونهاى بيوشيميايى از خون مادر كه شارئ

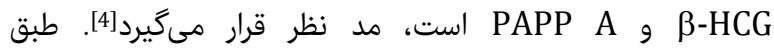

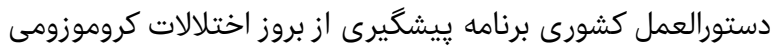
جنين در ايران، لزوم و اهميت استفاده از اين استانداردها تصريح برديح

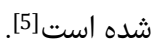

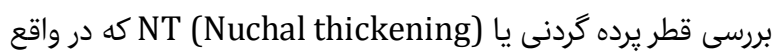
بيشترين اندازه منطقه شفاف بين يورست و بردئ بافت نرم روى ستون 


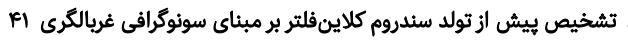

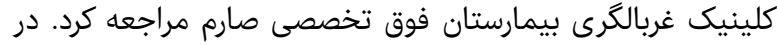

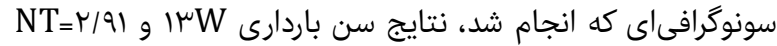

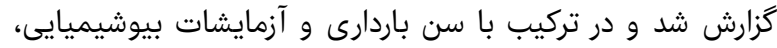

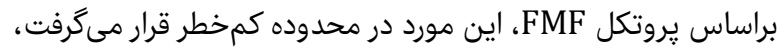

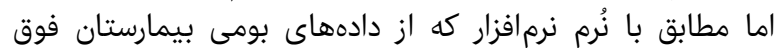

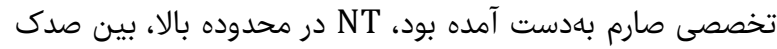

90 تا 99 قرار داشت (جدار بدول (1)
كونهاى انجام شود كه بر اهميت NT و لزوم انجام بررسىهاى بيشتر كروموزومى متمركز شود.

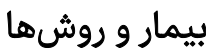

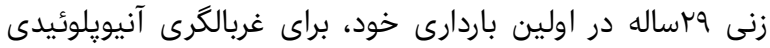

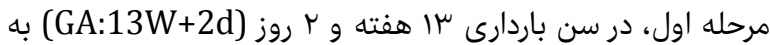

\begin{tabular}{|c|c|c|c|c|c|}
\hline 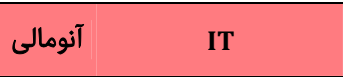 & $\begin{array}{c}\text { NT } \\
\text { (ميلىمتر) }\end{array}$ & NB & $\begin{array}{c}\text { CRL } \\
\text { (ميلىمتر) }\end{array}$ & $\begin{array}{c}\text { FHR } \\
\text { (ضربه/دقيقه) }\end{array}$ & نتايج \\
\hline \multirow[t]{4}{*}{ ديده شد (ترانس لوسنى ديده نشد } & $r / 91$ & |ديده شد & Vr & $10 \wedge$ & نتايج سونوگرافى مرحله اول \\
\hline & & & $\begin{array}{c}\text { PAPP A } \\
\text { ^/7IV/Lit } \\
\text { MOM : / /oOr }\end{array}$ & $\begin{array}{l}\text { Free } \beta \text {-HCG } \\
\text { q/OIV/Lit } \\
\text { MOM: •/rqr }\end{array}$ & يج آزمايش خون مادر \\
\hline & & & ميزان ريسك & سندروم & \\
\hline & & & $\begin{array}{l}\text { rrrE:1 } \\
\text { r...:I! }\end{array}$ & سندروم ادوارد (تريزومى شیانو (تريزومى) & 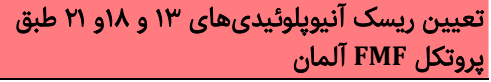 \\
\hline
\end{tabular}

صورتى كه NT بيشتر از / اميلىمتر و صدك بيشتر از 99\% باشد،

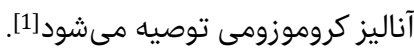

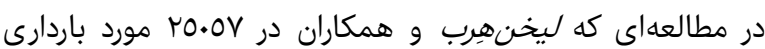

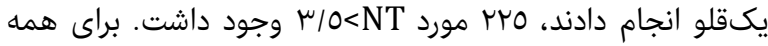

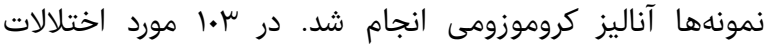

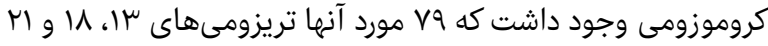

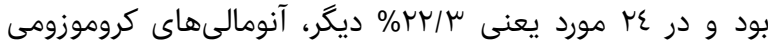
وجود داشت[16].

در متاآناليز انجامشده در طول يك ئ سال، نتيجه

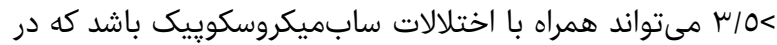

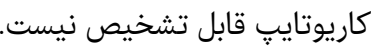

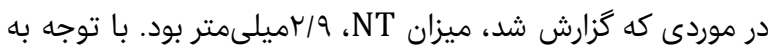

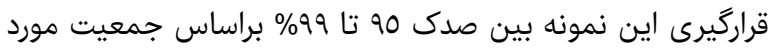

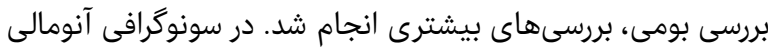

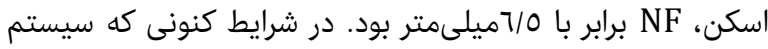

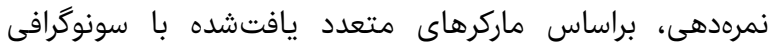

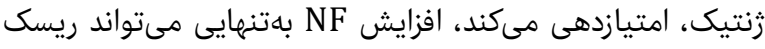

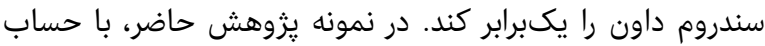

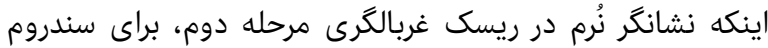

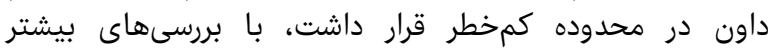

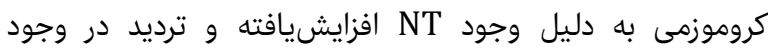
آنومالى، يى به وجود ناهنجارى كلاين فلتر برده شد.

\section{نتيجه گيرى}

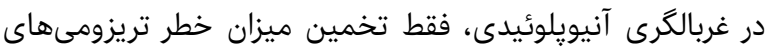

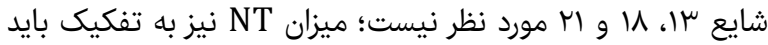

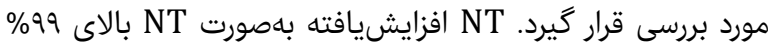

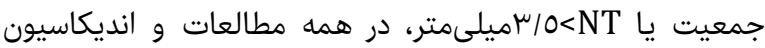

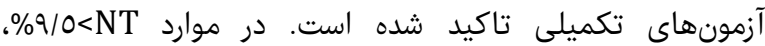

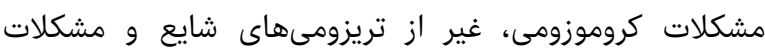

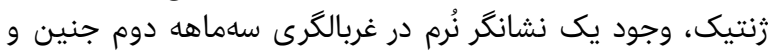

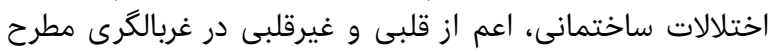

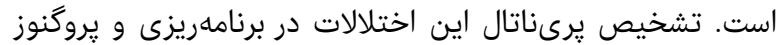

در غربالگرى مرحله دوم اين نمونه، آزمايش كوآدماركر (آزمايشات

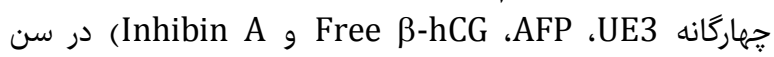

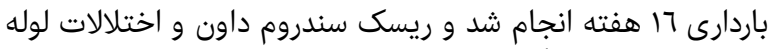

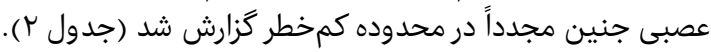

جدول Y) نتايج مراحل مختلف غربالگرى سهماهه دوم

\begin{tabular}{|c|c|c|c|}
\hline NF & آنومالى & $\begin{array}{c}\text { FHR } \\
\text { (ضربه/دقيقه) }\end{array}$ & نتايج \\
\hline ه/\$ميلىمتر & ديده نشد & $10 r$ & $\begin{array}{r}\text { نتايج سونوكرافى مرحله دوم } \\
\text { (GA=19W+rd) }\end{array}$ \\
\hline & ميزان ريسك & سندروم & \\
\hline & $\begin{array}{l}1: \Delta \ldots \\
1: \& \Delta . .\end{array}$ & 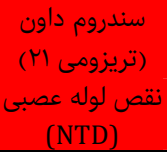 & 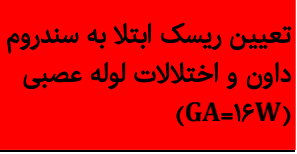 \\
\hline
\end{tabular}

طبق سونوگرافى، ساير قسمتها از نظر آنومالى نرمال بود و ساير

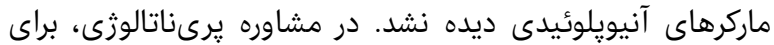

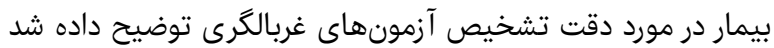

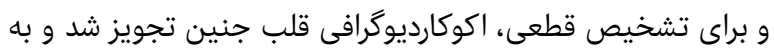

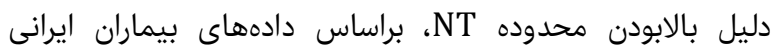
مراجعهكننده به بيمارستان فوق تخصصى محدى صارم، بررسى آمنيوسنتز

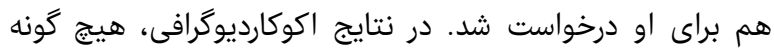

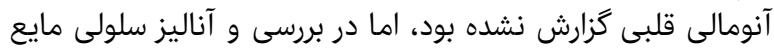

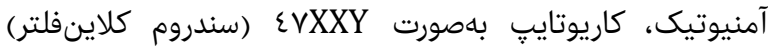

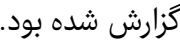

بحث

افزايش NT (بالاى صدك 99\% جمعيت يا بيشتر از / / اميلىمتر)

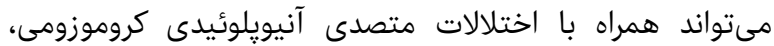

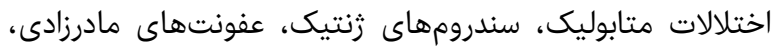

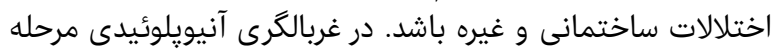
يك، براساس NT و ميزان PAPP-A و و

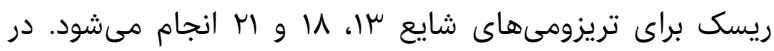


[Persian]

7- Piri S. Screening of chromosomal abnormalities in 1st and 2nd trimester of pregnancy: The role of fetal echocardiographic genetic sonography. J Iran Obstetr Gynecol. 2010;5(1):7-12. [Persian]

8- Driscoll DA, Gross SJ. Screening for fetal aneuploidy and neural tube defects. Genet Med. 2009;11(11):818-21.

9- Vladareanu R, Tutunaru D, Alexandru B, Veduta A, Zvanca M. Ultrasound in prenatal diagnosis of triploidy and Turner syndrome. Gynaecol Perinatol. 2006;15(4):192-201.

10- Zoppi M, Ibba R, Floris M, Monni G. Fetal nuchal translucency screening in 12495 pregnancies in Sardinia. Ultrasound Obstet Gynecol. 2001;18(6):649-51.

11- Spitczok von Brisinski I. Klinefelter syndrome: the commonest form of hypogonadism, but often overlooked or untreated. Psychological and neurological problems. Dtsch Arztebl Int. 2013;110(40):675.

12- Sanz-Cortés M, Raga F, Cuesta A, Claramunt R, Bonilla-Musoles F. Prenatally detected double trisomy: Klinefelter and Down syndrome. Prenat Diagn. 2006;26(11):1078-80.

13- Mishra SR, Bisht JS, Kumar M, Kumar S, Gaur K, Kumar R, et al. Double aneuploidy 48,XXY,+21 in a fetus with congenital abnormalities. Der Pharmacia Lettre. 2014;6(3):351-4.

14-Shu X, Zou C, Shen Z. Double aneuploidy 48, $\mathrm{XXY},+21$ associated with a aongenital aeart aefect in a neonate balkan. J Med Genet. 2013;16(2):8590.

15- Jo DG, Seo JT, Lee JS, Park SY, Kim JW. Klinefelter syndrome diagnosed by prenatal screening tests in high-risk groups. Korean J Urol. 2013;54(4):263-5.

16- Lichtenbelt KD, Alizadeh BZ, Scheffer PG, Stoutenbeek P, Schielen PC, Page-Christiaens LC, et al. Trends in the utilization of invasive prenatal diagnosis in the Netherlands during 2000-2009. Prenat Diagn. 2011;31(8):765-72.

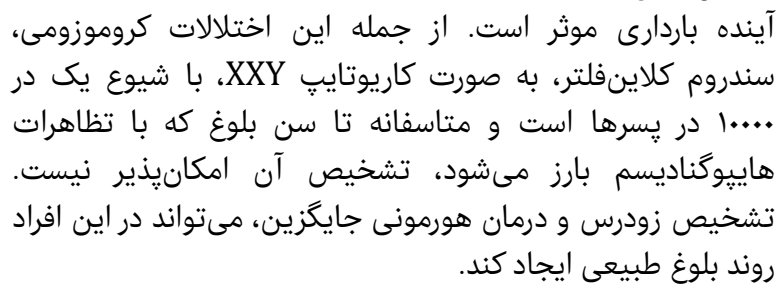

1- Nicolaides K. The 11-13+ 6 Weeks Scan. London: Fetal Medicine Foundation; 2004.

2- Karami F, Noori-Daloii M, Modarressi M. Noninvasive screening methods of down syndrome through maternal circulation. Novin Genetics. 2015;10(2):135-50 [Persian]

3- Miron P, Côté YP, Lambert J. Nuchal translucency thresholds in prenatal screening for Down syndrome and trisomy 18. J Obstet Gynaecol Can. 2009 Mar;31(3):227-235.

4- Palomaki GE, Lee JES, Canick JA, McDowell GA, Donnenfeld AE. Technical standards and guidelines: Prenatal screening for Down syndrome that includes first-trimester biochemistry and/or ultrasound measurements. Genet Med. 2009;11(9):669-81.

5- Islamic Republic of Iran Ministry of Health and Medical Education. Fetal chromosomal abnormalities prevent programs down syndrome [Online]. Tehran: Ministry of Health Medical Education; 2015 [Update 2016 May 10]. Available From: https://goo.gl/NvQ8Hz. [Persian]

6- Vallian Broojeni S. Prenatal diagnosis of genetic diseases. J Labratoratory Diagn. 2013(19):30-6. 